\title{
Values of applying white blood cell counts in the prognostic evaluation of resectable colorectal cancer
}

\author{
JING WU $^{1 *}$, XIN-XIN GE ${ }^{1 *}$, WENYU ZHU ${ }^{2 *}$, QIAOMING ZHI $^{3 *}$, MENG-DAN XU $^{1}$, WEIMING DUAN ${ }^{1}$, \\ KAI CHEN $^{1}$, FEI-RAN GONG ${ }^{4}$, MIN TAO ${ }^{1,5}$, LIU-MEI SHOU ${ }^{6}$, MENG-YAO WU ${ }^{1}$ and WEN-JIE WANG ${ }^{1,7}$ \\ ${ }^{1}$ Department of Oncology, The First Affiliated Hospital of Soochow University, Suzhou, Jiangsu 215006; \\ ${ }^{2}$ Department of Oncology, The Affiliated Changzhou No. 2 People's Hospital, Nanjing Medical University, Changzhou, \\ Jiangsu 213000; Departments of ${ }^{3}$ General Surgery and ${ }^{4}$ Hematology, The First Affiliated Hospital of Soochow University, \\ Suzhou, Jiangsu 215006; ${ }^{5}$ PREMED Key Laboratory for Precision Medicine, Soochow University, Suzhou, \\ Jiangsu 215021; ${ }^{6}$ Department of Oncology, The First Affiliated Hospital of Zhejiang Chinese \\ Medicine University, Hangzhou, Zhejiang 310006; ${ }^{7}$ Department of Radio-Oncology, The Affiliated \\ Suzhou Hospital of Nanjing Medical University, Suzhou, Jiangsu 215001, P.R. China
}

Received November 24, 2017; Accepted July 9, 2018

DOI: $10.3892 / \mathrm{mmr} .2019 .9844$

\begin{abstract}
The count and classification of white blood cells (WBCs) may be used as prognostic markers in certain types of cancer. The present study investigated the prognostic potential of the counts of WBCs, including lymphocytes (LYs), monocytes (MOs), neutrophils (NEs), eosinophils (EOs) and basophils (BAs), in the prognosis of resectable colorectal cancer. The present study recruited 153 resectable colorectal cancer cases retrospectively, which were pathologically confirmed. All patients were divided into two groups, according to the median value of LY (low LY, $\leq 1.632 \times 10^{9} / 1$ or high $\mathrm{LY},>1.632 \times 10^{9} / 1$ ), MO (low MO, $\leq 0.330 \times 10^{9} / 1$ or high MO, $>0.330 \times 10^{9} / 1$ ), $\mathrm{NE}$ (low NE, $\leq 3.600 \times 10^{9} / 1$ or high $\mathrm{NE},>3.600 \times 10^{9} / 1$ ), EO (low EO, $\leq 0.085 \times 10^{9} / 1$ or high EO, $>0.085 \times 10^{9} / 1$ ), BA (low $\mathrm{BA}, \leq 0.010 \times 10^{9} / 1$ or high $\mathrm{BA},>0.010 \times 10^{9} / 1$ ), or WBC (low $\mathrm{WBC}, \leq 5.780 \times 10^{9} / 1$ or high $\left.\mathrm{WBC},>5.780 \times 10^{9} / 1\right)$. To evaluate the alterations in WBC counts following surgery and adjuvant chemotherapy; all samples received oxiplatin and capecitabine (XELOX) for 6-8 cycles or 5-fluorouracil, leucovorin and oxaliplatin (mFOLFOX6) for 10-12 cycles. XELOX
\end{abstract}

Correspondence to: Dr Wen-Jie Wang, Department of Radio-Oncology, The Affiliated Suzhou Hospital of Nanjing Medical University, 26 Daoqian Road, Suzhou, Jiangsu 215001, P.R. China

E-mail: doctor.wjwang@gmail.com

Dr Meng-Yao Wu, Department of Oncology, The First Affiliated Hospital of Soochow University, 899 Pinghai Road, Suzhou, Jiangsu 215006, P.R. China

E-mail: mywu@suda.edu.cn

${ }^{*}$ Contributed equally

Key words: prognosis, overall survival, white blood cell, lymphocyte, neutrophil, eosinophil, basophil, colorectal cancer included oxaliplatin administered intravenously at a dose of $130 \mathrm{mg} / \mathrm{m}^{2}$ on day 1 and $850-1,250 \mathrm{mg} / \mathrm{m}^{2}$ capecitabine twice daily for days $1-14$, repeated every 3 weeks. mFOLFOX6 included oxaliplatin administered intravenously at a dose of $85 \mathrm{mg} / \mathrm{m}^{2}, 400 \mathrm{mg} / \mathrm{m}^{2}$ leucovorin and $400 \mathrm{mg} / \mathrm{m}^{2} 5-\mathrm{FU}$ on day 1 followed by $1,200 \mathrm{mg} / \mathrm{m}^{2} /$ days continuous infusion for 2 days (in total, $2,400 \mathrm{mg} / \mathrm{m}^{2}$ over $46-48 \mathrm{~h}$ ), repeated every 2 weeks. The present study investigated the post/pre-treatment of $\mathrm{LY}, \mathrm{MO}, \mathrm{NE}, \mathrm{EO}, \mathrm{BA}$ and $\mathrm{WBC}$ ratios ( $\leq 1$ indicated that LY, MO, NE, EO, BA and WBC counts were not increased following therapy; whereas, $>1$ suggested increased counts). Kaplan-Meier curves were constructed to demonstrate overall survival (OS). A multivariate and univariate logistic regression analyses model was employed to identify the independent risk factors. Low pre-treatment BA counts were associated with larger tumor size $(>5 \mathrm{~cm})$; pre-treatment BA levels were positively associated with OS. Surgery significantly decreased the count of BAs and increased the count of EOs; whereas, no effect was observed on LYs, MOs, NEs or WBCs. Adjuvant chemotherapy markedly decreased the counts of LY, NE and WBC; whereas, no notable effects on MOs, EOs or BAs were observed. Whole course treatment (surgery combined with adjuvant chemotherapy) significantly decreased the values of LY, NE and WBC; however, increased the value of EO; no effects on the MO or BA counts were observed. An increased post-/pre-treatment NE ratio suggested poorer prognosis. Multivariate Cox regression analysis revealed that sex, tumor size, pre-treatment BA count and the post-/pre-treatment NE ratio were independent prognostic factors affecting OS. The results of the present study suggested that the pre-treatment BA count and post-/pre-treatment NE ratio may be potential prognostic factors for resectable colorectal cancer.

\section{Introduction}

Colorectal cancer (CRC), which is the third most commonly diagnosed cancer, is the second and third leading cause of 
cancer-associated mortality in men and women, respectively (1). The prognosis of CRC is associated with the time of diagnosis, the depth of tumor invasion, metastasis to regional lymph nodes and distant metastasis (2). It is widely reported that surgical resection and post-operative adjuvant chemotherapy are the principal therapies used for patients with resectable CRC (3).

Inflammation is one of the most common pathological reactions in the body and is closely associated with tumorigenesis (4). The classification and the count of white blood cells (WBCs) may alter when inflammation occurs and may be associated with particular types of cancer, including bladder cancer (5), breast cancer (6) and prostate cancer (7). WBCs may be categorized into eosinophils (EOs), basophils (BAs), neutrophils (NEs), lymphocytes (LYs) and monocytes (MOs), based on the morphology of cells and nuclei (8). Among these, EOs serve important roles in allergic diseases and helminth infections (9). In addition, as multifunctional leukocytes, EOs have additionally been demonstrated to be involved in anticancer immunity (10). BAs, as the least abundant type of granulocytes, account for $<1 \%$ of circulating leukocytes and were first described as a type of immune cell responsible for immediate hypersensitivity (11).

Numerous previous studies have demonstrated the prognostic value of the NE-LY ratio (NLR) in patients with CRC $(12,13)$. The NLR reflects the activity of the innate (NEs) and adaptive (LYs) aspects of the immune system (14); however, it is unclear whether components of the NLR contribute equally to the prognostic value (14). Peripheral blood LYs have a vital role in the tumor immune response, via the recognition and elimination of tumor cells (15). NEs are crucial for tumor progression by releasing various pro-angiogenic factors, including vascular endothelial growth factor, interleukin (IL)-8 and matrix metalloproteinase 9 (14).

In addition, MOs are markers of inflammation and associated with tumor prognosis (16). The aim of the present study was to investigate whether the count and classifications of WBCs serve as markers with high sensitivity and as prognostic factors in patients with resectable CRC.

\section{Patients and methods}

Subjects and inclusion criteria. The present study was conducted as a retrospective investigation of patients with CRC that had been referred to The First Affiliated Hospital of Soochow University (Suzhou, China) between June 2006 and July 2017. Approval for the study was granted by the Medical Ethics Committees of The First Affiliated Hospital of Soochow University. Written informed consent was obtained from all patients. The inclusion criteria were as follows: i) Those with histologically or cytologically confirmed resectable colorectal cancer; ii) age 18-70 years; iii) Karnofsky performance status score of $\geq 70$ (17); iv) those with a predicted survival of $\geq 6$ months; v) either naive to anti-tumor treatment or the postoperative adjuvant chemotherapy was performed $\geq 6$ months after the last dose of chemotherapy; and vi) those who met the following laboratory criteria: WBCs $\geq 4.0 \times 10^{9} / 1$; absolute NE count $\geq 2.0 \times 10^{9} / 1$; and $\mathrm{PLT} \geq 100 \times 10^{9} / 1$. The exclusion criterion was that the patient failed to complete adjuvant chemotherapy following surgery. Clinical and pathological records of all the patients participating in the study were reviewed periodically, the first follow-up was 3 months following adjuvant chemotherapy and the last follow-up was conducted in July 2017.

In total, 153 patients with CRC were included in the present study. All cases were confirmed by surgery and pathological analysis. In the present study, all patients underwent surgery and adjuvant chemotherapy. Patient characteristics are presented in Table I. The median age of the 153 patients was 56-years old (range of 27-85 years); 71 patients were male and 82 were female. Pathological staging of the patients was conducted according to the tumor-node-metastases classification system and classified via the American Joint Committee on Cancer (AJCC) recommendations (18); prognostic analyses were performed regarding overall survival (OS).

Blood samples. Peripheral venous blood $(5-7 \mathrm{ml})$ was collected into a sterile EDTA tube; patients fasted for $8 \mathrm{~h}$ and samples were obtained from the elbow veins between 6:30 and 7:30 a.m. in order to standardize the known impact of circulating hormones (circadian rhythm) on the number and subtype distribution of the various WBC indices. LY, MO, NE, EO, $\mathrm{BA}$ and $\mathrm{WBC}$ were analyzed within $30 \mathrm{~min}$ following collection using a hematology analyzer (Sysmex XE-2100; Sysmex Corporation, Kobe, Japan). LY, MO, NE, EO, BA and WBC levels were recorded. The patients were divided into two groups according to the median values of LY, MO, NE, EO, BA or WBC. The post-/pre-treatment ratios were defined as the rate of pre-treatment blood parameters values and the corresponding values obtained following therapy. Blood samples from all patients were obtained 1 month following surgery and 3 months following adjuvant chemotherapy. In the present study, all patients underwent surgery and adjuvant chemotherapy. Surgery was performed for conventional mesocolic excision or complete mesocolic excision in patients with colon cancer, conventional mesocolic excision or total mesocolic excision in patients with colon cancer. Adjuvant chemotherapy included oxiplatin and capecitabine (XELOX) for 6-8 cycles or 5-fluorouracil, leucovorin and oxaliplatin (mFOLFOX6) for 10-12 cycles. XELOX included oxaliplatin administered intravenously at a dose of $130 \mathrm{mg} / \mathrm{m}^{2}$ on day 1 and $850-1,250 \mathrm{mg} / \mathrm{m}^{2}$ capecitabine twice daily for days $1-14$, repeated every 3 weeks. mFOLFOX6 included oxaliplatin administered intravenously at a dose of $85 \mathrm{mg} / \mathrm{m}^{2}, 400 \mathrm{mg} / \mathrm{m}^{2}$ leucovorin and $400 \mathrm{mg} / \mathrm{m}^{2} 5-\mathrm{FU}$ on day 1 followed by $1,200 \mathrm{mg} / \mathrm{m}^{2} /$ days continuous infusion for 2 days (in total, 2,400 mg/m² over 46-48 h), repeated every 2 weeks.

Evaluation. Computed tomography scans were performed for the assessment of response to treatment every 2 months with all treatments lasting for 6 months, and evaluated according to the criteria of Response Evaluation Criteria in Solid Tumors 1.1 (19).

Follow-up. Survival time was measured from the date of chemotherapy administration until mortality or the last clinical evaluation. The prognostic analyses were performed regarding OS, which was defined as the duration between the date of diagnosis and mortality due to any cause.

Statistical analysis. All statistical analyses were performed using SPSS 19.0 software (IBM Corp., Armonk, NY, USA). For the analysis of survival data, Kaplan-Meier curves were 


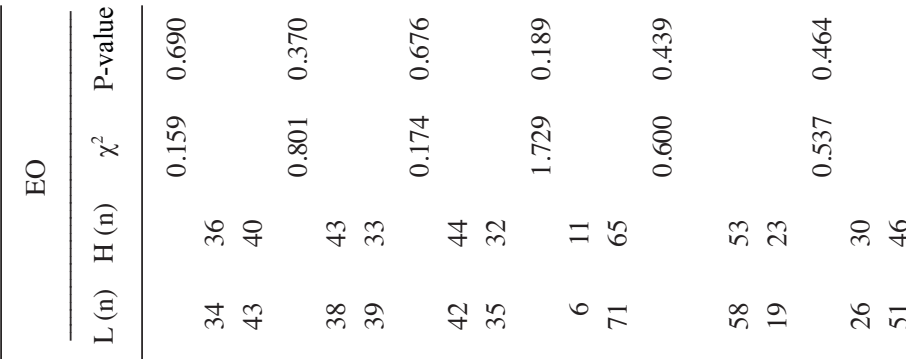

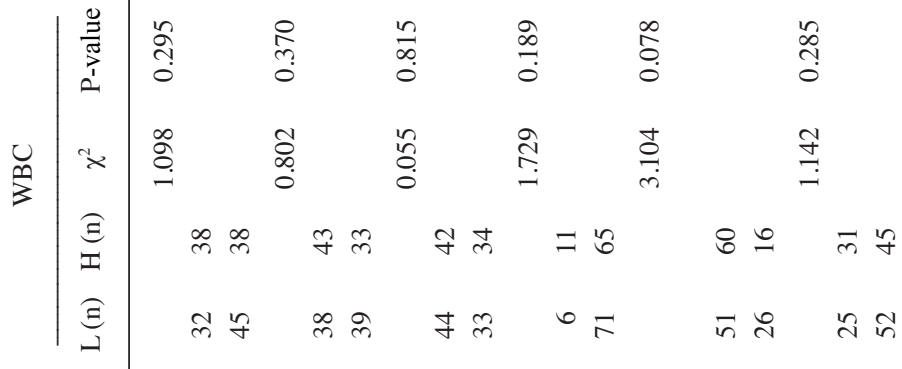

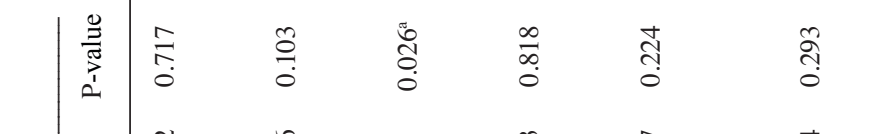



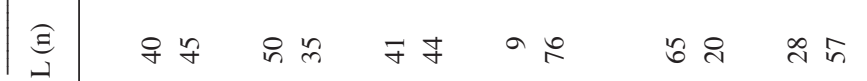



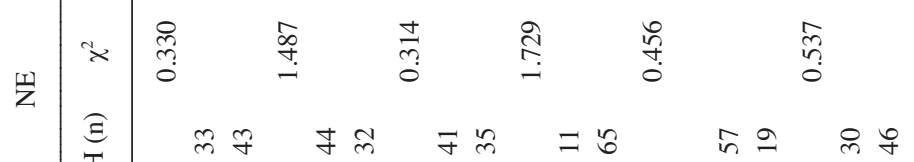

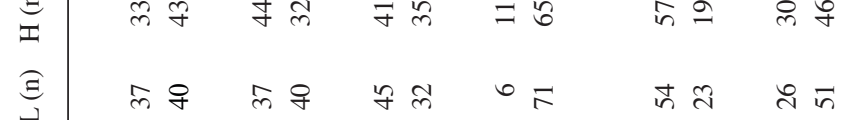

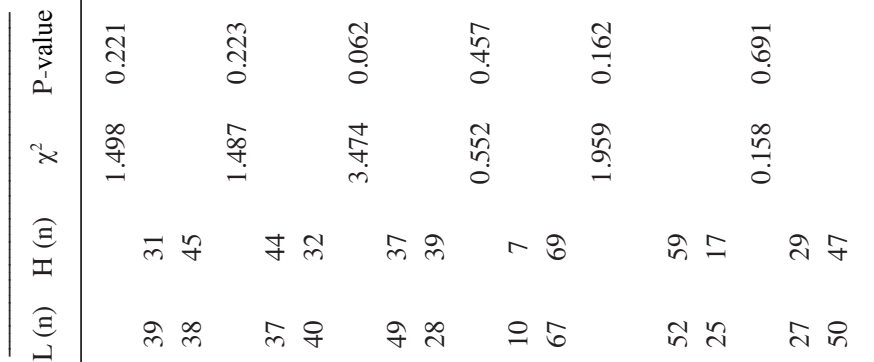

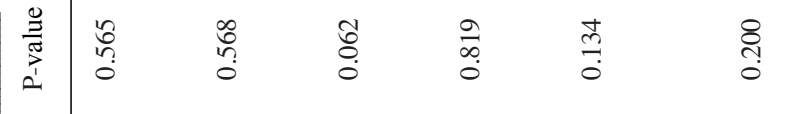

离

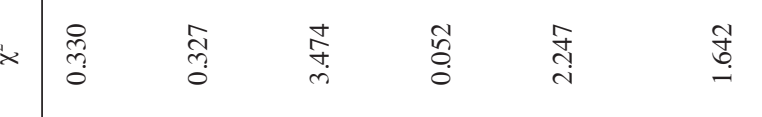

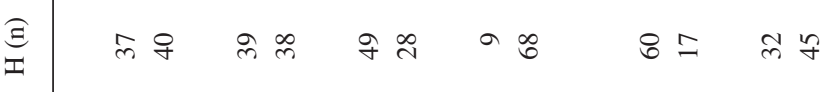

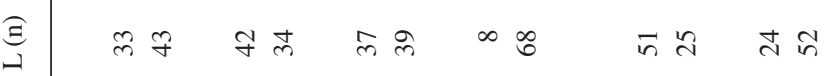





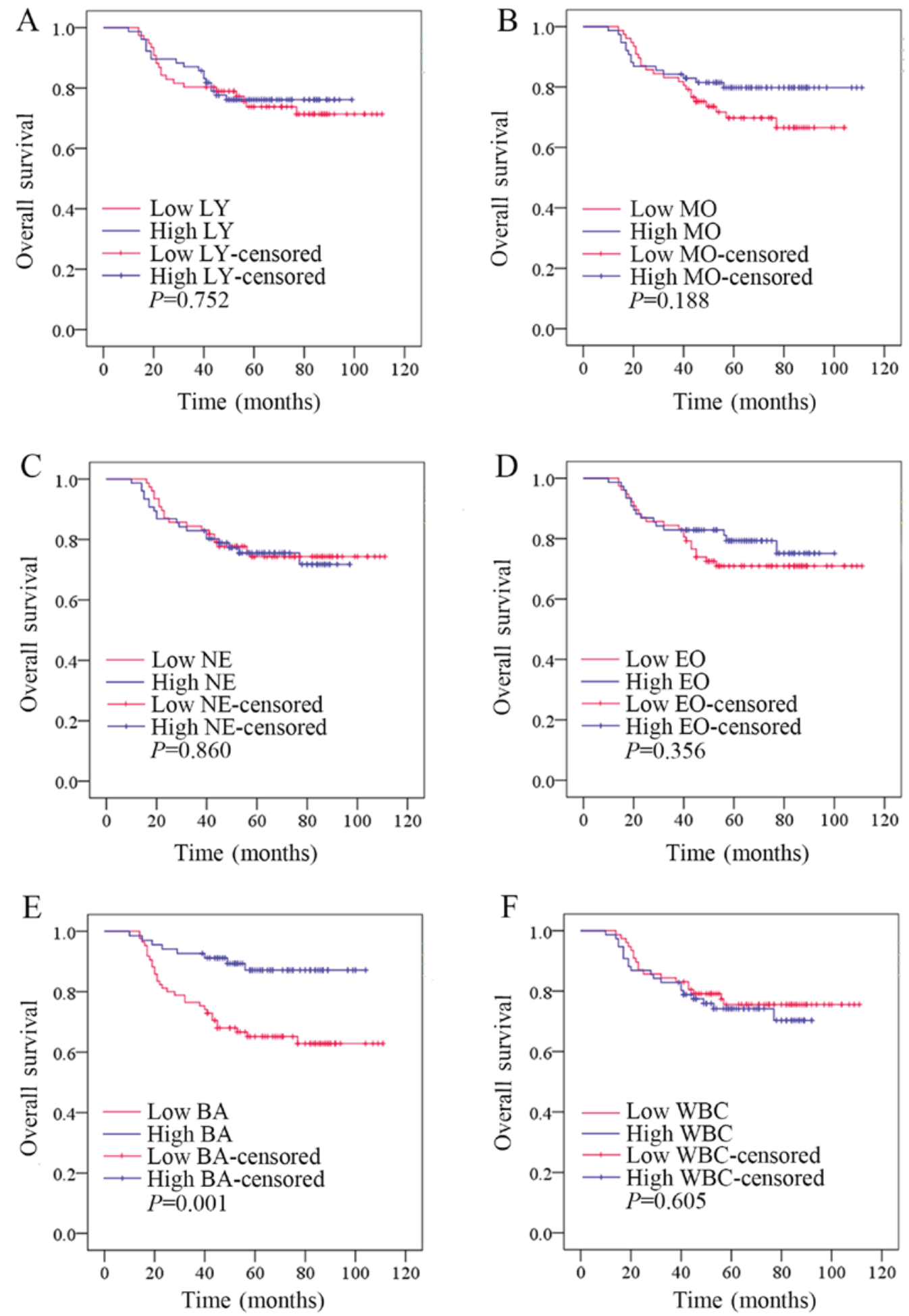

Figure 1. Association between the status of pre-treatment WBC counts and patient outcomes. (A) OS according to LY. (B) OS according to MO. (C) OS according to NE. (D) OS according to EO. (E) OS according to BA. (F) OS according to WBC. n=153. BA, basophil; EO, eosinophil; LY, lymphocyte; MO, monocyte; NE, neutrophil; OS, overall survival; WBC, white blood cell.

constructed and statistical analysis was conducted with a log-rank test. The associations between the status of blood parameters and clinicopathological features were analyzed using $\chi^{2}$ test. The associations between alterations in the status of the blood parameters and surgery or chemotherapy were assessed by Paired Samples t-test. As the BA values were relatively small compared with other indicators, the P-value demonstrated statistical significance; however, it was difficult to illustrate these differences on graphs. Multivariate and univariate logistic regression analyses were conducted to identify the independent risk factors associated with CRC using a backwards elimination technique, which involves including all the independent variables into the equation and gradually eliminating the non-statistical independent variables, to derive a potentially suitable set of predictors (20). OS was defined as the time from the initiation of surgery to the patient succumbing to any cause. Numerical data 

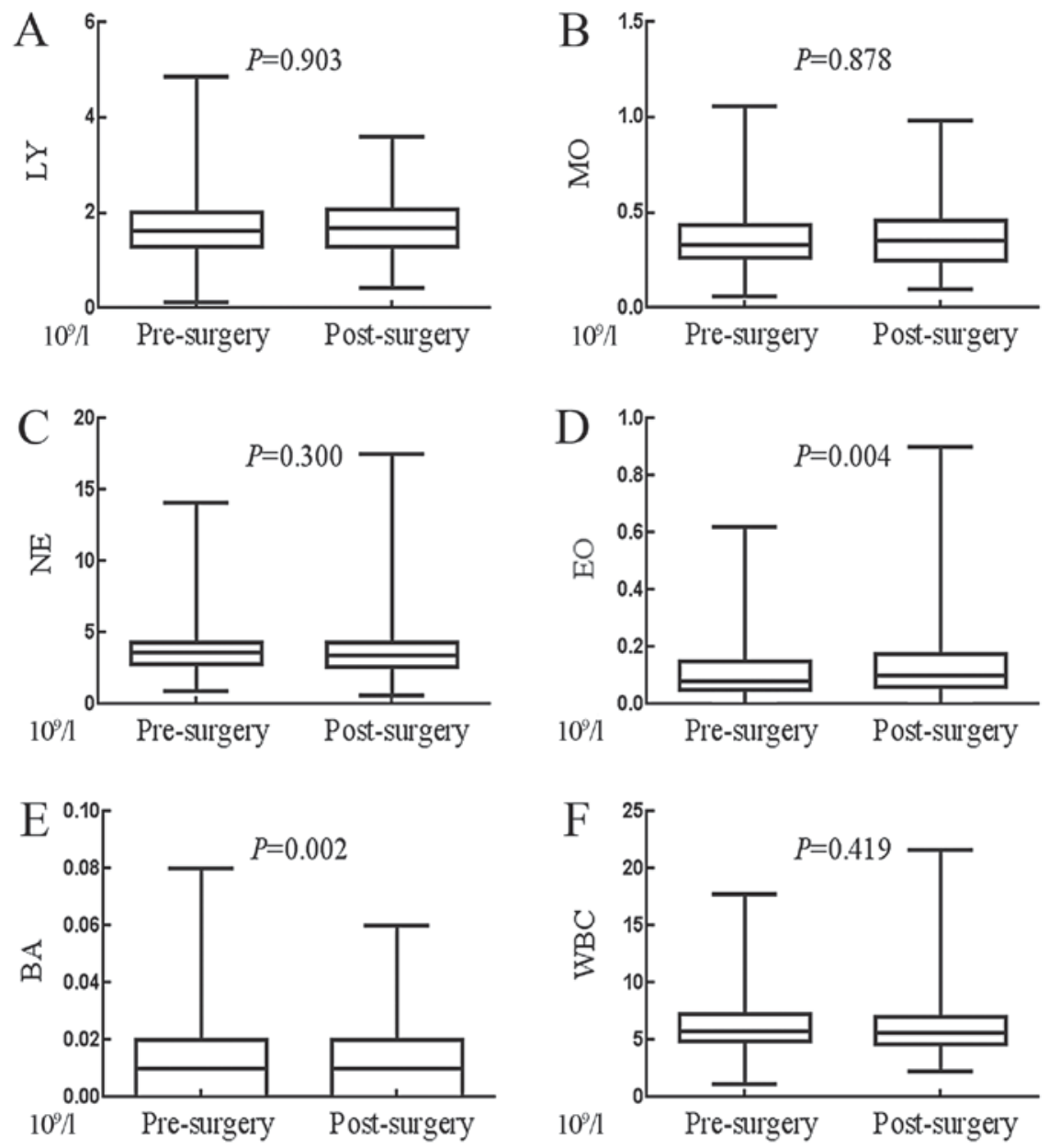

Figure 2. Association between alterations in the status of WBC counts and surgery. (A) Surgery had no effect on LY counts. (B) Surgery had no effect on MO counts. (C) Surgery decreased NE counts. (D) Surgery increased EO counts. (E) Surgery decreased BA counts. (F) Surgery had no effect on WBC counts. $\mathrm{n}=153$. BA, basophil; EO, eosinophil; LY, lymphocyte; MO, monocyte; NE, neutrophil; WBC, white blood cell.

are presented as the mean \pm standard error. $\mathrm{P}<0.05$ was considered to indicate a statistically significant difference.

\section{Results}

Pre-treatment BA levels are associated with the outcomes of patients with $C R C$. The patients were divided into two groups according to the median values of LY (low LY, $\leq 1.632 \times 10^{9} / 1$; or high $\mathrm{LY},>1.632 \times 10^{9} / 1$ ), MO (low $\mathrm{MO}, \leq 0.330 \times 10^{9} / 1$; or high $\mathrm{MO},>0.330 \times 10^{9} / 1$ ), NE (low NE, $\leq 3.600 \times 10^{9} / 1$; or high $\mathrm{NE},>3.600 \times 10^{9} / 1$ ), EO (low EO, $\leq 0.085 \times 10^{9} / 1$; or high $\left.\mathrm{EO},>0.085 \times 10^{9} / 1\right)$, BA (low BA, $\leq 0.010 \times 10^{9} / 1$; or high $\mathrm{BA}$, $\left.>0.010 \times 10^{9} / 1\right)$, or WBC (low WBC, $\leq 5.780 \times 10^{9} / 1$; or high $\mathrm{WBC}$, $\left.>5.780 \times 10^{9} / 1\right)$. Kaplan-Meier plots were produced to determine the association between LY, MO, NE, EO, BA and WBC status and OS (Fig. 1). The mean OS of the high LY group was 82.438 months [95\% confidence interval (CI), 75.635-89.241], while that of the low LY group was 88.670 months $(95 \% \mathrm{CI}$, 80.209-97.130; $\mathrm{P}=0.752)$. The mean OS was 93.644 months $(95 \%$ CI, 85.712-101.576) in the high MO group and 81.539 months (95\% CI, 73.795-89.282) in the low MO group ( $\mathrm{P}=0.188)$. The mean OS of the high NE group was 79.186 months (95\% CI, 72.153-86.220), while that of the low NE group was 90.586 months (95\% CI, 82.554-98.618; $\mathrm{P}=0.860)$. The mean OS was 83.867 months (95\% CI, 76.806-90.928) in the high
EO group and 87.778 months (95\% CI, 79.469-96.088) in the low EO group $(\mathrm{P}=0.356)$. The mean $\mathrm{OS}$ of the high $\mathrm{BA}$ group was 94.753 months (95\% CI, 88.688-100.817), while that of the low BA group was 81.506 months (95\% CI, 72.919-90.092; $\mathrm{P}=0.001)$. The mean OS was 74.923 months (95\% CI, 68.366-81.479) in the high WBC group and 91.433 months (95\% CI, 83.461-81.479) in the low WBC group ( $\mathrm{P}=0.605)$. Therefore, higher pre-treatment BA levels were associated with improved prognosis; however, the pre-treatment levels of LY, $\mathrm{MO}, \mathrm{NE}, \mathrm{EO}$ or WBC were not significantly associated with OS.

Effects of surgery on the counts of WBCs. The effects of surgery on the counts of the various types of WBCs are presented in Fig. 2. The median value of LY was $1.630 \times 10^{9} / 1$

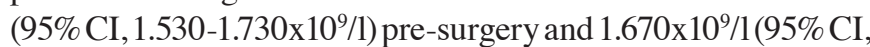
$\left.1.540-1.780 \times 10^{9} / 1\right)$ post-surgery $(\mathrm{P}=0.903)$. The median value of $\mathrm{MO}$ was $0.330 \times 10^{9} / 1\left(95 \% \mathrm{CI}, 0.3100 .360 \times 10^{9} / 1\right)$ pre-surgery and $0.350 \times 10^{9} / 1\left(95 \% \mathrm{CI}, 0.320-0.370 \times 10^{9} / 1\right)$ post-surgery $(\mathrm{P}=0.878)$. The median value of $\mathrm{NE}$ was $3.600 \times 10^{9} / 1(95 \% \mathrm{CI}$, $\left.3.410-3.790 \times 10^{9} / 1\right)$ pre-surgery and $3.400 \times 10^{9} / 1(95 \% \mathrm{CI}$, $\left.3.170-3.800 \times 10^{9} / 1\right)$ post-surgery $(\mathrm{P}=0.300)$. The median value of EO was $0.080 \times 10^{9} / 1\left(95 \%\right.$ CI, $\left.0.080-0.100 \times 10^{9} / 1\right)$ pre-surgery and $0.100 \times 10^{9} / 1\left(95 \% \mathrm{CI}, 0.090-0.110 \times 10^{9} / 1\right)$ post-surgery $(\mathrm{P}=0.004)$. The median value of $\mathrm{BA}$ was $0.010 \times 10^{9} / 1$ 



Figure 3. Association between alterations in the number of WBCs and adjuvant chemotherapy. (A) Chemotherapy decreased the levels of LY. (B) Chemotherapy had no effect on the levels of MO. (C) Chemotherapy decreased the levels of NE. (D) Chemotherapy had no effect on the levels of EO. (E) Chemotherapy had no effect on the value of BA. (F) Chemotherapy decreased the value of WBC. n=153. BA, basophil; EO, eosinophil; LY, lymphocyte; MO, monocyte; NE, neutrophil; WBC, white blood cell.

(95\% CI, $\left.0.010-0.010 \times 10^{9} / 1\right)$ pre-surgery and $0.010 \times 10^{9} / 1$ $\left(95 \% \mathrm{CI}, 0.000-0.010 \times 10^{9} / 1\right)$ post-surgery $(\mathrm{P}=0.002)$. The median value of WBC was $5.780 \times 10^{9} / 1\left(95 \%\right.$ CI, $\left.5.480-5.940 \times 10^{9} / 1\right)$

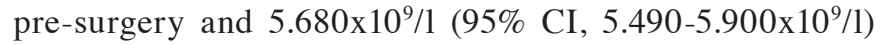
post-surgery $(\mathrm{P}=0.419)$. The results suggested that surgery increased the levels of EO and BA; however, exhibited no effect on the values of LY, MO, NE or WBC.

Effects of adjuvant chemotherapy on the counts of WBCs. The effects of adjuvant chemotherapy on the counts of WBCs are presented in Fig. 3. The median value of LYs was $1.670 \times 10^{9} / 1$ (95\% CI, 1.540-1.780x10\%/l) pre-adjuvant chemotherapy and $1.470 \times 10^{9} / 1\left(95 \% \mathrm{CI}, 1.370-1.610 \times 10^{9} / \mathrm{l}\right)$ post-adjuvant chemotherapy $(\mathrm{P}=0.007)$. The median value of $\mathrm{MO}$ was $0.350 \times 10^{9} / 1$ (95\% CI, 0.320-0.370 $\left.\times 10^{9} / 1\right)$ pre-adjuvant chemotherapy and $0.320 \times 10^{9} / 1\left(95 \%\right.$ CI, $\left.0.290-0.340 \times 10^{9} / 1\right)$ post-adjuvant chemotherapy $(\mathrm{P}=0.376)$. The median value of $\mathrm{NE}$ was $3.400 \times 10^{9} / 1$ (95\% CI, 3.170-3.800 $\left.\times 10^{9} / 1\right)$ pre-adjuvant chemotherapy and $3.010 \times 10^{9} / 1$ (95\% CI, 2.880-3.340x10/1) post-adjuvant chemotherapy $(\mathrm{P}=0.011)$. The median value of $\mathrm{EO}$ was $0.100 \times 10^{9} / 1$ (95\% CI, 0.090-0.110x10\%/) pre-adjuvant chemotherapy and $0.090 \times 10^{9} / 1$ (95\% CI, $\left.0.070-0.120 \times 10^{9} / 1\right)$ post-adjuvant chemotherapy $(\mathrm{P}=0.689)$. The median value of $\mathrm{BA}$ was $0.010 \times 10^{9} / 1$ (95\% CI, 0.000-0.010x10 $/ 1$ ) pre-adjuvant chemotherapy and
$0.016 \times 10^{9} / 1$ (95\% CI, $\left.0.011-0.023 \times 10^{9} / 1\right)$ post-adjuvant chemotherapy $(\mathrm{P}=0.117)$. The median value of $\mathrm{WBC}$ was $5.680 \times 10^{9} / 1$ (95\% CI, 5.490-5.900x10\%1) pre-adjuvant chemotherapy and $5.280 \times 10^{9} / 1\left(95 \%\right.$ CI, $\left.4.790-5.700 \times 10^{9} / 1\right)$ post-adjuvant chemotherapy $(\mathrm{P}=0.002)$. Therefore, adjuvant chemotherapy may have decreased the levels of LY, NE and WBC; however, exhibited no significant impact on the values of $\mathrm{MO}, \mathrm{EO}$ or $\mathrm{BA}$.

Effects of whole course treatment on the counts of WBCs. The impacts of whole course treatment (surgery and adjuvant chemotherapy) on the counts of WBCs are presented in Fig. 4. The median value of $\mathrm{LY}$ was $1.630 \times 10^{9} / \mathrm{l}\left(95 \% \mathrm{CI}, 1.530-1.730 \times 10^{\%} / \mathrm{l}\right)$ pre-treatment and $1.470 \times 10^{9} / 1\left(95 \% \mathrm{CI}, 1.370-1.610 \times 10^{9} / \mathrm{l}\right)$ post-treatment $(\mathrm{P}=0.017)$. The median value of $\mathrm{MO}$ was $0.330 \times 10^{9} / 1\left(95 \%\right.$ CI, $\left.0.310-0.360 \times 10^{9} / 1\right)$ pre-treatment and $0.320 \times 10^{9} / 1\left(95 \% \mathrm{CI}, 0.290-0.340 \times 10^{9} / 1\right)$ post-treatment $(\mathrm{P}=0.474)$. The median value of $\mathrm{NE}$ was $3.600 \times 10^{9} / 1(95 \% \mathrm{CI}$, $\left.3.410-3.790 \times 10^{9} / 1\right)$ pre-treatment and $3.010 \times 10^{9} / 1(95 \% \mathrm{CI}$, 2.880-3.340 $\left.\times 10^{9} / 1\right)$ post-treatment $(\mathrm{P}<0.001)$. The median value of $\mathrm{EO}$ was $0.085 \times 10^{9} / 1\left(95 \% \mathrm{CI}, 0.080-0.100 \times 10^{9} / 1\right)$ pre-treatment and $0.090 \times 10^{9} / 1$ (95\% CI, $\left.0.070-0.120 \times 10^{9} / 1\right)$ post-treatment $(\mathrm{P}=0.045)$. The median value of $\mathrm{BA}$ was $0.010 \times 10^{\%} / 1\left(95 \%\right.$ CI, $\left.0.010-0.010 \times 10^{9} / 1\right)$ pre-treatment and $0.016 \times 10^{9} / 1\left(95 \%\right.$ CI, $\left.0.011-0.023 \times 10^{9} / 1\right)$ post-treatment 

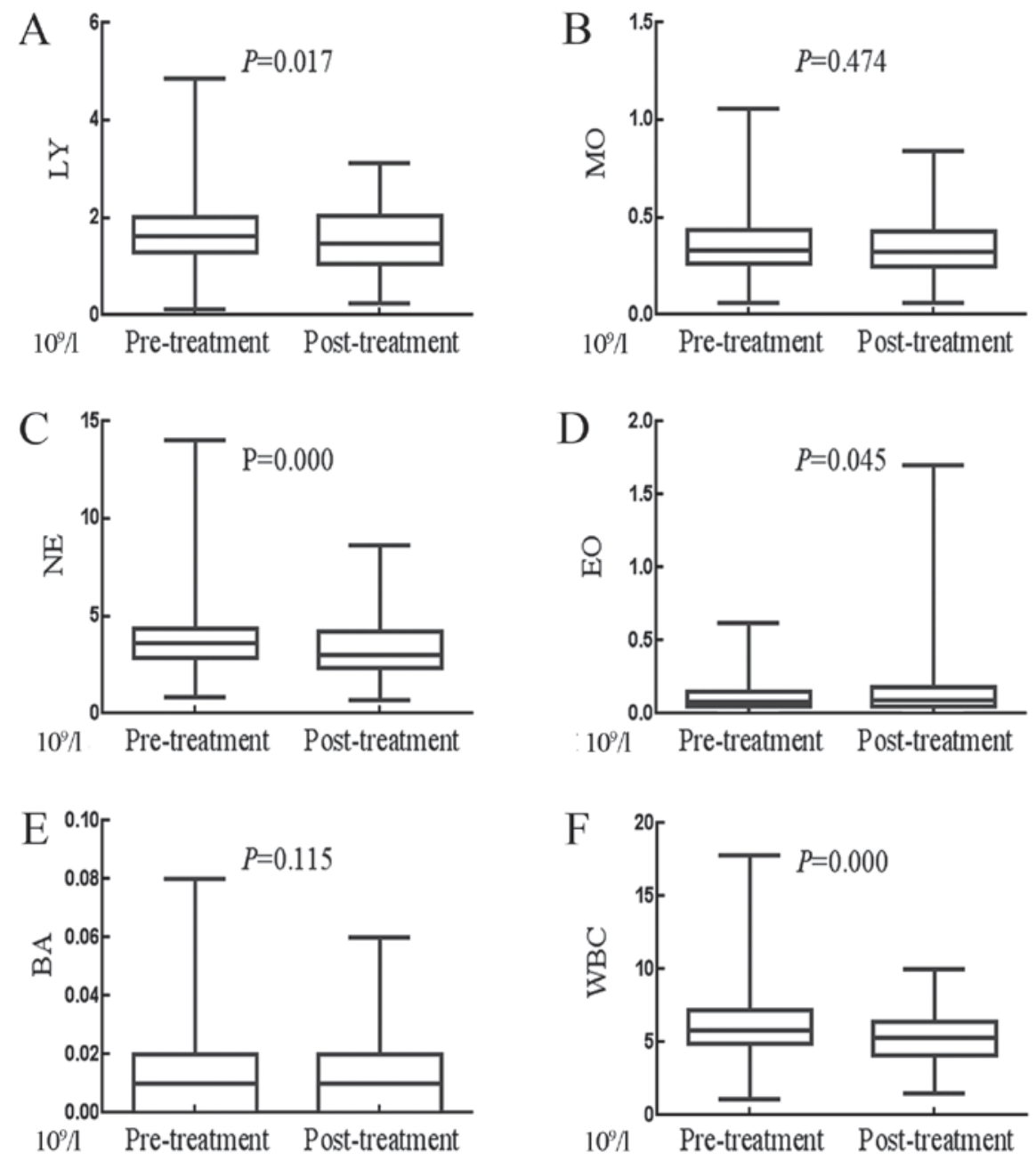

Figure 4. Association between alterations in the status WBC counts and whole course treatment. (A) Whole course treatment decreased the levels of LY. (B) Whole course treatment had no effect on the levels of MO. (C) Whole course treatment decreased the NE counts. (D) Whole course treatment increased the EO count. (E) Whole course of treatment had no influence on the BA counts. (F) Whole course of treatment decreased the value of WBC. $n=153$. BA, basophil; EO, eosinophil; LY, lymphocyte; MO, monocyte; NE, neutrophil; WBC, white blood cell.

$(\mathrm{P}=0.115)$. The median value of $\mathrm{WBC}$ was $5.780 \times 10^{9} / 1(95 \% \mathrm{CI}$,

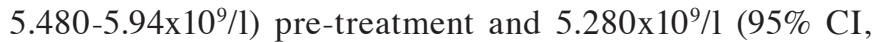
4.790-5.700x $\left.10^{9} / 1\right)$ post-treatment $(\mathrm{P}<0.001)$. Therefore, whole course treatment significantly decreased the values of LY, NE and $\mathrm{WBC}$; however increased the value of $\mathrm{EO}$, whereas no significant effects on the levels of MO or BA were observed.

Alterations in NE levels following whole course treatment are associated with the outcomes of patients with resectable CRC. Kaplan-Meier plots were used to determine the effects of alterations of LY, MO, NE, EO, BA and WBC status on OS (Fig. 5). The mean OS of patients with increased LY levels following whole course treatment was 87.782 months (95\% CI, 79.055-96.508); whereas, that of the unaltered LY group was 86.107 months (95\% CI, 78.763-93.450; $\mathrm{P}=0.516$ ). The mean OS of patients with increased MO levels following whole course treatment was 84.173 months (95\% CI, 77.050-91.297); whereas, that of the unaltered MO group was 88.142 months (95\% CI, 80.062-96.222; $\mathrm{P}=0.528$ ). The mean OS of patients with increased NE levels following whole course of treatment was 70.872 months (95\% CI, 60.825-80.920), while that of the unaltered NE group was 95.664 months (95\% CI, 89.379-101.948; $\mathrm{P}=0.005)$. The mean OS of patients with increased EO levels following whole course treatment was 84.245 months (95\% CI, 75.092-93.398), while that of the unaltered EO group was 89.443 months $(95 \%$ CI, 82.799-96.087; $\mathrm{P}=0.066$ ). The mean OS of patients with increased BA levels following whole course of treatment was 79.730 months (95\% CI, 69.770-89.691), while that of the unaltered BA group was 92.373 months (95\% CI, 85.453-99.293; $\mathrm{P}=0.205)$. The mean OS of patients with increased WBC levels following whole course of treatment was 76.339 months $(95 \% \mathrm{CI}$, 66.959-85.720), while that of the unaltered WBC group was 93.180 months (95\% CI, 86.355-100.005; $\mathrm{P}=0.137)$. Therefore, the patients with increased NE levels post-therapy had decreased survival; however, alterations in LY, MO, EO, BA and WBC levels exhibited no marked effects on OS.

Prognostic factors for resectable CRC. Univariate analyses demonstrated that sex [women; hazard ratio (HR) 2.911; 95\% CI, 1.413-5.998; $\mathrm{P}=0.004]$, tumor size ( $>5 \mathrm{~cm}$; HR, 2.613; 95\% CI, 1.351-5.054; $\mathrm{P}=0.004)$, lymphonodus metastasis (N2; HR, 2.197; 95\% CI, 1.153-4.187; P=0.017), AJCC stage (III; HR, 2.408; 95\% CI, 1.103-5.254; $\mathrm{P}=0.027)$, low pre-treatment BA levels (HR, 3.333; 95\% CI, 1.524-7.246; $\mathrm{P}=0.003$ ) and increased post-/pre-treatment $\mathrm{NE}$ ratio (HR, 2.410; 95\% CI, 1.275-4.554; $\mathrm{P}=0.007$ ) were significant risk factors for a poor 



Figure 5. Association between alterations in the status of post-/pre-treatment counts of WBCs and the outcomes. (A) OS according to alterations in LY. (B) OS according to alterations in MO. (C) OS according to alterations in NE. (D) OS according to alterations in EO. (E) OS according to alterations in BA. (F) OS according to alterations in WBC. $\mathrm{n}=153$. BA, basophil; EO, eosinophil; LY, lymphocyte; MO, monocyte; NE, neutrophil; OS, overall survival; WBC, white blood cell.

prognosis (Table II). In multivariate analysis, sex (women; HR, 3.503; 95\% CI, 1.620-7.576; $\mathrm{P}=0.001)$, tumor size $(>5 \mathrm{~cm}$; HR, 2.329; 95\% CI, 1.101-4.930; $\mathrm{P}=0.027)$, low pre-treatment BA (HR, 3.984; 95\% CI, 1.751-9.009; P=0.001) and increased post-/pre-treatment NE ratio (HR, 2.444; 95\% CI, 1.240-4.814; $\mathrm{P}=0.010)$ were observed to be independently associated with poor survival.

\section{Discussion}

WBCs, produced in the bone marrow, are indicators of inflammation, trauma, allergies, leukemia or infections;
WBC infiltration is frequently observed in numerous types of cancer (21). WBCs may be classified into five forms, LY, MO, $\mathrm{NE}, \mathrm{EO}$ and BA, each with various different roles.

In the present study, the LY count was not associated with prognosis; however, previous studies have demonstrated that the LY level is a prognostic factor for non-small-cell lung cancer, gallbladder carcinoma, cervical cancer and hepatocellular carcinoma $(15,22-25)$. This may be due to tumor-infiltrating LYs, which activate $\mathrm{CD}^{+} \mathrm{T}$ LYs by inducing the apoptosis of tumor cells (26). In addition, the primary influencing factors of the anti-tumor immune response are $\mathrm{T}$ LYs (27). Tumor-infiltrating LYs are core components of the 
Table II. Univariate and multivariate logistic regression analysis of risk factors.

\begin{tabular}{|c|c|c|c|c|}
\hline \multirow[b]{3}{*}{ Risk factors } & \multicolumn{4}{|c|}{ Overall survival } \\
\hline & \multicolumn{2}{|c|}{ Univariate analysis } & \multicolumn{2}{|c|}{ Multivariate analysis } \\
\hline & OR $(95 \% \mathrm{CI})$ & P-value & OR $(95 \% \mathrm{CI})$ & P-value \\
\hline Sex (women or men) & $2.911(1.413-5.998)$ & $0.004^{\mathrm{a}}$ & $3.503(1.620-7.576)$ & $0.001^{\mathrm{a}}$ \\
\hline Age ( $>56$ years or $\leq 56$ years $)$ & $1.003(0.974-1.034)$ & 0.825 & - & - \\
\hline Tumor size $(>5$ or $\leq 5 \mathrm{~cm})$ & $2.613(1.351-5.054)$ & $0.004^{\mathrm{a}}$ & $2.329(1.101-4.930)$ & $0.027^{\mathrm{b}}$ \\
\hline Depth of invasion (T3-4 or T1-2) & $2.591(0.624-10.77)$ & 0.190 & - & - \\
\hline Lymphonodus metastasis (N2 or N0-1) & $2.197(1.153-4.187)$ & $0.017^{\mathrm{b}}$ & $2.100(0.955-4.619)$ & 0.065 \\
\hline American Joint Committee on Cancer stage (III or I-II) & $2.408(1.103-5.254)$ & $0.027^{\mathrm{b}}$ & $1.328(0.524-3.366)$ & 0.550 \\
\hline Pre-treatment LY level $\left(>1.632 \times 10^{9} / 1\right.$ or $\left.\leq 1.632 \times 10^{9} / 1\right)$ & $0.903(0.477-1.708)$ & 0.753 & - & - \\
\hline Pre-treatment MO level $\left(>0.330 \times 10^{9} / 1\right.$ or $\left.\leq 0.330 \times 10^{9} / 1\right)$ & $0.649(0.338-1.244)$ & 0.193 & - & - \\
\hline Pre-treatment NE level $\left(>3.600 \times 10^{9} / 1\right.$ or $\left.\leq 3.600 \times 10^{9} / 1\right)$ & $1.059(0.560-2.000)$ & 0.860 & - & - \\
\hline Pre-treatment EO level $\left(>0.085 \times 10^{9} / 1\right.$ or $\left.\leq 0.085 \times 10^{9} / 1\right)$ & $0.740(0.388-1.409)$ & 0.359 & - & - \\
\hline Pre-treatment BA level $\left(\leq 0.010 \times 10^{9} / 1\right.$ or $\left.>0.010 \times 10^{9} / 1\right)$ & $3.333(1.524-7.246)$ & $0.003^{\mathrm{a}}$ & $3.984(1.751-9.009)$ & $0.001^{\mathrm{a}}$ \\
\hline Pre-treatment WBC level $\left(>5.780 \times 10^{9} / 1\right.$ or $\left.\leq 5.780 \times 10^{9} / 1\right)$ & $1.182(0.625-2.235)$ & 0.606 & - & - \\
\hline Post-/pre-treatment LY ratio $(>1$ or $\leq 1)$ & $1.234(0.652-2.336)$ & 0.518 & - & - \\
\hline Post-/pre-treatment MO ratio $(>1$ or $\leq 1)$ & $0.812(0.423-1.557)$ & 0.530 & - & - \\
\hline Post-/pre-treatment NE ratio $(>1$ or $\leq 1)$ & $2.410(1.275-4.554)$ & $0.007^{\mathrm{a}}$ & $2.444(1.240-4.814)$ & $0.010^{\mathrm{b}}$ \\
\hline Post-/pre-treatment EO ratio $(>1$ or $\leq 1)$ & $1.820(0.949-3.489)$ & 0.071 & - & - \\
\hline Post-/pre-treatment BA ratio $(>1$ or $\leq 1)$ & $1.511(0.793-2.880)$ & 0.209 & - & - \\
\hline Post-/pre-treatment WBC ratio $(>1$ or $\leq 1)$ & $1.615(0.852-3.063)$ & 0.142 & - & - \\
\hline
\end{tabular}

${ }^{\mathrm{a}} \mathrm{P}<0.01$; ${ }^{\mathrm{P}}<0.05$; BA, basophil; CI, confidence interval; LY, lymphocyte; EO, eosinophil; MO, monocyte; NE, neutrophil; OR, odds ratio; WBC, white blood cell.

immune response to tumors, with cytotoxic T LYs serving a key role in the eradication of tumors; whereas, regulating T cells may inhibit the immune response $(28,29)$.

Numerous mechanisms mediate the association between elevated NE counts and poor prognosis. Cancer cells are involved in the production of NEs via the release of myeloid growth factors (30). In feedback mechanisms, elevated NE levels promote angiogenesis by secreting factors, including vascular endothelial growth factor, interleukin (IL)- 8 and matrix metallopeptidase, which contribute to the progression of cancer (31). Additionally, numerous previous studies have revealed an important association between the NLR and survival in a variety of malignancies, including CRC (32-34). In the present study, it was demonstrated that the NE count may be reduced in response to chemotherapy. As surgery exhibited no effect on NE status, the contribution of reduced NE levels may be primarily associated with chemotherapy. In addition, based on alterations in individual NE levels, the increased post-/pre-treatment NE ratio was associated with reduced OS, and demonstrated to be an independent prognostic marker of poor survival via univariate and multivariate analyses. This suggested that NEs may be a poor marker of prognosis for patients with resectable CRC. As the LY count was not determined to be associated with prognosis, the present study proposed that the association between NLR and survival of patients with resectable CRC maybe primarily due to the association between $\mathrm{NE}$ and prognosis.

MOs have been proposed as a prognostic factor in numerous types of cancer, including cervical cancer, melanoma and colorectal cancer (35); cancer-associated inflammation contributes to tumor proliferation, angiogenesis, metastasis and drug resistance (36). MOs, as a part of systemic inflammation responses, serve a critical role in tumorigenesis (37). Tumor-associated macrophages (TAMs) originate from MOs and have an important role in the tumor microenvironment (37). TAMs exhibit pro-tumoral activity by accelerating tumor proliferation and metastasis, immunosuppression and angiogenesis (38-40). Chan et al (41) reported that myeloid-derived suppressor cells, which possess immunosuppressive activity, are a subset of circulating leucocytes. High MO counts have been associated with the poor prognosis of cancer via elevated monocytic myeloid-derived suppressor cell counts (41); however, in the present study, surgery and/or chemotherapy did not affect the MO count. In addition, MOs were not observed to be associated with prognosis.

The prognostic role of EO in patients with cancer remains controversial (10). Previous studies have demonstrated that EO was associated with a better prognosis in a number of types of cancer, including laryngeal and colon cancer $(42,43)$. Two mechanisms may be involved in the anti-tumor effects of EOs. EOs are recruited into tumor tissues, and establish contact with tumor cells, inducing a tumoricidal effect (10). EOs may additionally secrete granule proteins and cytokines, including EO cationic protein, EO-derived neurotoxin, tumor necrosis factor- $\alpha, 2 \mathrm{~B} 4$ and cross-linked 2B4, which are involved in tumor cell death $(42,44)$. Conversely, previous studies have demonstrated that EOs had no association or were negatively associated with the prognosis of patients with stage III and IV laryngeal squamous cell carcinoma or nodular sclerosis Hodgkin's disease, respectively $(45,46)$. The present study revealed that EOs were not associated with age, sex, tumor size, depth of invasion, lymphonodus metastasis or AJCC stage; however, surgery increased the levels of 
EO. The EO count was not observed to be associated with the prognosis of patients with resectable CRC in the present study.

BA is the least abundant type of WBC in the circulation and has not been studied extensively (47). A number of previous studies have investigated the prognostic potential of BA in cancer progression (47-49). As a type of immune cell, BA has been demonstrated to be negatively associated with tumor prognosis in pancreatic cancer (47). De Monte et al (47) revealed that the infiltration of BAs in tumor-draining lymph nodes was associated with the Th2/Th1 cell ratio in tumors and was responsible for the presence of IL- 4 in pancreatic cancer. In addition, the counts of circulating BAs in patients with non-small cell lung cancer were higher compared with in healthy individuals (50). Furthermore, in a mouse model of metastatic breast cancer generated by implanting 4T1 cells into the mammary fat pads, Wang et al (21) reported that circulating BAs were negatively associated with the number of lung metastases. In the present study, the BA count was negatively associated with tumor size and was proposed to be an independent prognostic indicator for CRC; no effects on age, sex, depth of invasion, lymphonodus metastasis or AJCC stage were observed. Additionally, the present study reported that the pre-treatment BA count was positively associated with OS, suggesting BA may be a prognostic indicator of resectable CRC. Additionally, surgery may increase the BA count; however, differences in the BA levels between the pre- and post-surgery groups were difficult to determine as the BA count was low and was not distinguishable.

The limitations of the present study include its retrospective design, data obtained from a single center and an insufficient number of cases for study. Future studies may aim to investigate larger sample sizes and use multiple approaches.

In conclusion, the results of the present study revealed that a low pre-treatment BA count and an increased post-/pre-treatment NE ratio were positively associated with poorer outcomes in patients with resectable CRC. These noninvasive, simple and low-cost biomarkers may serve as useful prognostic indicators for patients with resectable CRC.

\section{Acknowledgements}

Not applicable.

\section{Funding}

The present study was supported by the National Natural Science Foundation of China (grant nos. 81472296, 81602091, 81402176, 81402093, 81272542 and 81200369), the Six Major Talent Peak Project of Jiangsu Province (grant no. 2015-WSN-022; China), the Project of Invigorating Health Care through Science, Technology and Education, Jiangsu Provincial Medical Youth Talent (grant no. QNRC2016709; China), the Project of Jiangsu Provincial Commission of Health and Family Planning (grant no. H201518; China), the Science and Education for Health Foundation of Suzhou for Youth (grant no. kjxw2015003; China) and the Science and Technology Project Foundation of Suzhou (grant nos. SYS201464 and SYS201504; China).

\section{Availability of data and materials}

The datasets used or analyzed during the current study are available from the corresponding author on reasonable request.

\section{Authors' contributions}

WJW and MYW made substantial contributions to the conception and design of the study; JW, QZ, WZ and XXG revised the manuscript critically for important intellectual content and acquired the data; MDX, WD, KC, FRG, MT and LMS analyzed and interpreted the data for the present study. All authors have read and approved the final version of the manuscript.

\section{Ethics approval and consent to participate}

The present study was approved by the Medical Ethics Committees of The First Affiliated Hospital of Soochow University (Suzhou, China). Written informed consent was obtained from all patients.

\section{Patient consent for publication}

Not applicable.

\section{Competing interests}

The authors declare that they have no competing interests.

\section{References}

1. Siegel RL, Miller KD and Jemal A: Cancer Statistics, 2017. CA Cancer J Clin 67: 7-30, 2017.

2. Gennari L, Doci R and Rossetti C: Prognostic factors in colorectal cancer. Hepatogastroenterology 47: 310-314, 2000.

3. Kim MN, Kang JM, Yang JI, Kim BK, Im JP, Kim SG, Jung HC, Song IS and Kim JS: Clinical features and prognosis of early colorectal cancer treated by endoscopic mucosal resection. J Gastroenterol Hepatol 26: 1619-1625, 2011.

4. Hussain SP and Harris CC: Inflammation and cancer: An ancient link with novel potentials. Int J Cancer 121: 2373-2380, 2007.

5. Cimen HI, Halis F, Saglam HS and Gokce A: Can neutrophil to lymphocyte ratio predict lamina propria invasion in patients with non muscle invasive bladder cancer? Int Braz J Urol 43: 67-72, 2017.

6. Sun H, Yin CQ, Liu Q, Wang F and Yuan CH: Clinical signi fi cance of routine blood test-associated inflammatory index in breast cancer patients. Med Sci Monit 23: 5090-5095, 2017.

7. Arthur R, Williams R, Garmo H, Holmberg L, Stattin P, Malmström H, Lambe M, Hammar N, Walldius G, Robinsson D, et al: Serum inflammatory markers in relation to prostate cancer severity and death in the Swedish AMORIS study. Int J Cancer 142: 2254-2262, 2018.

8. SuMC,Cheng CY and Wang PC: A neural-network-based approach to white blood cell classification. ScientificWorldJournal 2014: 796371, 2014.

9. Spergel J and Aceves SS: Allergic components of eosinophilic esophagitis. J Allergy Clin Immunol 142: 1-8, 2018.

10. Cao C, Gu Y, Zhu C, Palmai-Pallag T, Lan F, Chen Z, Li W, Shen $\mathrm{H}$ and Ying S: Potential roles of eosinophils in cancer therapy: Epidemiological studies, experimental models, and clinical pathology. Recent Pat Anticancer Drug Discov 9: 241-248, 2014.

11. Siracusa MC, Kim BS, Spergel JM and Artis D: Basophils and allergic inflammation. J Allergy Clin Immunol 132: 789-801, 2013.

12. Guthrie GJ, Roxburgh CS, Farhan-Alanie OM, Horgan PG and McMillan DC: Comparison of the prognostic value of longitudinal measurements of systemic inflammation in patients undergoing curative resection of colorectal cancer. Br J Cancer 109: 24-28, 2013. 
13. Zhang J, Zhang HY, Li J, Shao XY and Zhang CX: The elevated NLR, PLR and PLT may predict the prognosis of patients with colorectal cancer: A systematic review and meta-analysis. Oncotarget 8: 68837-68846, 2017.

14. Watt DG, Martin JC, Park JH, Horgan PG and McMillan DC: Neutrophil count is the most important prognostic component of the differential white cell count in patients undergoing elective surgery for colorectal cancer. Am J Surg 210: 24-30, 2015

15. Iwase R, Shiba H, Haruki K, Fujiwara Y, Furukawa K, Futagawa Y, Wakiyama S, Misawa T and Yanaga K: Post-operative lymphocyte count may predict the outcome of radical resection for gallbladder carcinoma. Anticancer Res 33: 3439-3444, 2013.

16. Shen SL, Fu SJ, Huang XQ, Chen B, Kuang M, Li SQ, Hua YP, Liang LJ and Peng BG: Elevated preoperative peripheral blood monocyte count predicts poor prognosis for hepatocellular carcinoma after curative resection. BMC Cancer 14: 744, 2014.

17. Wang F, Liu ZY, Xia YY, Zhou C, Shen XM, Li XL, Han SG, Zheng Y, Mao ZQ, Gong FR, et al: Changes in neutrophil/lymphocyte and platelet/lymphocyte ratios after chemotherapy correlate with chemotherapy response and prediction of prognosis in patients with unresectable gastric cancer. Oncol Lett 10: 3411-3418, 2015.

18. Edge SB and Compton CC: The American Joint Committee on Cancer: The 7th edition of the AJCC cancer staging manual and the future of TNM. Ann Surg Oncol 17: 1471-1474, 2010.

19. Eisenhauer EA, Therasse P, Bogaerts J, Schwartz LH, Sargent D, Ford R, Dancey J, Arbuck S, Gwyther S, Mooney M, et al: New response evaluation criteria in solid tumours: Revised RECIST guideline (version 1.1). Eur J Cancer 45: 228-247, 2009.

20. Hosmer DW Jr and Lemeshow S: Applied Logistic Regression, Second Edition, 2000

21. Wang C, Chen YG, Gao JL, Lyu GY, Su J, Zhang QI, Ji X, Yan JZ, Qiu QL, Zhang YL, et al: Low local blood perfusion, high white blood cell and high platelet count are associated with primary tumor growth and lung metastasis in a 4T1 mouse breast cancer metastasis model. Oncol Lett 10: 754-760, 2015.

22. Zhang J, Huang SH, Li H, Li Y, Chen XL, Zhang WQ, Chen HG and Gu LJ: Preoperative lymphocyte count is a favorable prognostic factor of disease-free survival in non-small-cell lung cancer. Med Oncol 30: 352, 2013.

23. Wang M, Li C, Wen TF, Peng W and Chen LP: Postoperative low absolute lymphocyte counts may predict poor outcomes of hepatocellular carcinoma after liver resection. Chin Med J (Engl) 129: 536-541, 2016.

24. Lee YY, Choi CH, Sung CO, Do IG, Hub SJ, Kim HJ, Kim TJ, Lee JW, Bae DS and Kim BG: Clinical significance of changes in peripheral lymphocyte count after surgery in early cervical cancer. Gynecol Oncol 127: 107-113, 2012.

25. Nagai S, Abouljoud MS, Kazimi M, Brown KA, Moonka D and Yoshida A: Peritransplant lymphopenia is a novel prognostic factor in recurrence of hepatocellular carcinoma after liver transplantation. Transplantation 97: 694-701, 2014.

26. Gooden MJ, de Bock GH, Leffers N, Daemen T and Nijman HW: The prognostic influence of tumour-infiltrating lymphocytes in cancer: A systematic review with meta-analysis. Br J Cancer 105: 93-103, 2011

27. Yu P and Fu YX: Tumor-infiltrating T lymphocytes: Friends or foes? Lab Invest 86: 231-245, 2006.

28. Fu J, Xu D, Liu Z, Shi M, Zhao P, Fu B, Zhang Z, Yang H, Zhang $\mathrm{H}$, Zhou $\mathrm{C}$, et al: Increased regulatory $\mathrm{T}$ cells correlate with CD8 T-cell impairment and poor survival in hepatocellular carcinoma patients. Gastroenterology 132: 2328-2339, 2007.

29. Unitt E, Marshall A, Gelson W, Rushbrook SM, Davies S, Vowler SL, Morris LS, Coleman N and Alexander GJ: Tumour lymphocytic infiltrate and recurrence of hepatocellular carcinoma following liver transplantation. J Hepatol 45: 246-253, 2006.

30. Li QQ, Lu ZH, Yang L, Lu M, Zhang XT, Li J, Zhou J, Wang XC, Gong JF, Gao J, et al: Neutrophil count and the inflammation-based glasgow prognostic score predict survival in patients with advanced gastric cancer receiving first-line chemotherapy. Asian Pac J Cancer Prev 15: 945-950, 2014

31. Guthrie GJ, Charles KA, Roxburgh CS, Horgan PG, McMillan DC and Clarke SJ: The systemic inflammation-based neutrophil-lymphocyte ratio: Experience in patients with cancer. Crit Rev Oncol Hematol 88: 218-230, 2013.
32. Yamanaka T, Matsumoto S, Teramukai S, Ishiwata R, Nagai Y and Fukushima M: The baseline ratio of neutrophils to lymphocytes is associated with patient prognosis in advanced gastric cancer. Oncology 73: 215-220, 2007

33. Fox P, Hudson M, Brown C, Lord S, Gebski V, De Souza P and Lee CK: Markers of systemic inflammation predict survival in patients with advanced renal cell cancer. Br J Cancer 109: 147-153, 2013.

34. Kishi Y, Kopetz S, Chun YS, Palavecino M, Abdalla EK and Vauthey JN: Blood neutrophil-to-lymphocyte ratio predicts survival in patients with colorectal liver metastases treated with systemic chemotherapy. Ann Surg Oncol 16: 614-622, 2009.

35. Lee YY, Choi CH, Sung CO, Do IG, Huh S, Song T, Kim MK, Kim HJ, Kim TJ, Lee JW, et al: Prognostic value of pre-treatment circulating monocyte count in patients with cervical cancer: Comparison with SCC-Ag level. Gynecol Oncol 124: 92-97, 2012.

36. Qi Q, Geng Y, Sun M, Wang P and Chen Z: Clinical implications of systemic inflammatory response markers as independent prognostic factors for advanced pancreatic cancer. Pancreatology 15 : $145-150,2015$

37. Mantovani A, Sica A and Locati M: New vistas on macrophage differentiation and activation. Eur J Immunol 37: 14-16, 2007.

38. Pollard JW: Macrophages define the invasive microenvironment in breast cancer. J Leukoc Biol 84: 623-630, 2008.

39. De Palma M and Naldini L: Angiopoietin-2 TIEs up macrophages in tumor angiogenesis. Clin Cancer Res 17: 5226-5232, 2011.

40. Mitchem JB, Brennan DJ, Knolhoff BL, Belt BA, Zhu Y, Sanford DE, Belaygorod L, Carpenter D, Collins L, Piwnica-Worms D, et al: Targeting tumor-infiltrating macrophages decreases tumor-initiating cells, relieves immunosuppression, and improves chemotherapeutic responses. Cancer Res 73: 1128-1141, 2013

41. Chan JC, Chan DL, Diakos CI, Engel A, Pavlakis N, Gill A and Clarke SJ: The lymphocyte-to-monocyte ratio is a superior predictor of overall survival in comparison to established biomarkers of resectable colorectal cancer. Ann Surg 265: 539-546, 2017.

42. Legrand F, Driss V, Delbeke M, Loiseau S, Hermann E, Dombrowicz D and Capron M: Human eosinophils exert TNF- $\alpha$ and granzyme A-mediated tumoricidal activity toward colon carcinoma cells. J Immunol 185: 7443-7451, 2010.

43. Thompson AC, Bradley PJ and Griffin NR: Tumor-associated tissue eosinophilia and long-term prognosis for carcinoma of the larynx. Am J Surg 168: 469-471, 1994.

44. Munitz A, Bachelet I, Fraenkel S, Katz G, Mandelboim O, Simon HU, Moretta L, Colonna M and Levi-Schaffer F: 2B4 (CD244) is expressed and functional on human eosinophils. J Immunol 174: 110-118, 2005.

45. Sassler AM, McClatchey KD, Wolf GT and Fisher SG: Eosinophilic infiltration in advanced laryngeal squamous cell carcinoma. Veterans Administration Laryngeal Cooperative Study Group. Laryngoscope 105: 413-416, 1995.

46. von Wasielewski R, Seth S, Franklin J, Fischer R, Hübner K, Hansmann ML, Diehl V and Georgii A: Tissue eosinophilia correlates strongly with poor prognosis in nodular sclerosing Hodgkin's disease, allowing for known prognostic factors. Blood 95: 1207-1213, 2000

47. De Monte L, Wörmann S, Brunetto E, Heltai S, Magliacane G, Reni M, Paganoni AM, Recalde H, Mondino A, Falconi M, et al: Basophil recruitment into tumor-draining lymph nodes correlates with Th2 inflammation and reduced survival in pancreatic cancer patients. Cancer Res 76: 1792-1803, 2016.

48. Cihan YB, Arslan A, Cetindag MF and Mutlu H: Lack of prognostic value of blood parameters in patients receiving adjuvant radiotherapy for breast cancer. Asian Pac J Cancer Prev 15: 4225-4231, 2014

49. White WM: Geochemistry: Portrait of Earth's coming of age. Nature 485: 452-453, 2012.

50. Anthony HM: Blood basophils in lung cancer. Br J Cancer 45 : 209-216, 1982. 